\title{
Neurocognitive impairment in type 2 diabetes: evidence for shared genetic aetiology
}

\author{
Josephine Mollon ${ }^{1}$ (D) - Joanne E. Curran ${ }^{2,3}$. Samuel R. Mathias ${ }^{1}$ • Emma E. M. Knowles ${ }^{1}$ • Phoebe Carlisle ${ }^{4}$. \\ Peter T. Fox ${ }^{5}$ - Rene L. Olvera ${ }^{5,6}$ • Harald H. H. Göring ${ }^{2,3}$ - Amanda Rodrigue ${ }^{1}$ - Laura Almasy ${ }^{7}$ - Ravi Duggirala ${ }^{2,3}$. \\ John Blangero ${ }^{2,3} \cdot$ David C. Glahn ${ }^{1,4}$
}

Received: 1 October 2019 / Accepted: 15 January 2020 / Published online: 4 February 2020

(C) Springer-Verlag GmbH Germany, part of Springer Nature 2020

\begin{abstract}
Aims/hypothesis Type 2 diabetes is associated with cognitive impairments, but it is unclear whether common genetic factors influence both type 2 diabetes risk and cognition.

Methods Using data from 1892 Mexican-American individuals from extended pedigrees, including 402 with type 2 diabetes, we examined possible pleiotropy between type 2 diabetes and cognitive functioning, as measured by a comprehensive neuropsychological test battery.

Results Negative phenotypic correlations $\left(\rho_{p}\right)$ were observed between type 2 diabetes and measures of attention (Continuous Performance Test [CPT d']: $\rho_{p}=-0.143, p=0.001$ ), verbal memory (California Verbal Learning Test [CVLT] recall: $\rho_{p}=-0.111$, $p=0.004$ ) and face memory (Penn Face Memory Test [PFMT]: $\rho_{p}=-0.127, p=0.002$; PFMT Delayed: $\rho_{p}=-0.148, p=2 \times 10^{-4}$ ), replicating findings of cognitive impairment in type 2 diabetes. Negative genetic correlations $\left(\rho_{g}\right)$ were also observed between type 2 diabetes and measures of attention (CPT d': $\rho_{g}=-0.401, p=0.001$ ), working memory (digit span backward test: $\rho_{g}=-0.380, p=0.005$ ), and face memory (PFMT: $\rho_{g}=-0.476, p=2 \times 10^{-4}$; PFMT Delayed: $\rho_{g}=-0.376, p=0.005$ ), suggesting that the same genetic factors underlying risk for type 2 diabetes also influence poor cognitive performance in these domains. Performance in these domains was also associated with type 2 diabetes risk using an endophenotype ranking value approach. Specifically, on measures of attention (CPT d': $\beta=$ $-0.219, p=0.005$ ), working memory (digit span backward: $\beta=-0.326, p=0.035)$, and face memory (PFMT: $\beta=-0.171, p=0.023$; PFMT Delayed: $\beta=-0.215, p=0.005$ ), individuals with type 2 diabetes showed the lowest performance, while unaffected/unrelated individuals showed the highest performance, and those related to an individual with type 2 diabetes performed at an intermediate level. Conclusions/interpretation These findings suggest that cognitive impairment may be a useful endophenotype of type 2 diabetes and, therefore, help to elucidate the pathophysiological underpinnings of this chronic disease.

Data availability The data analysed in this study is available in dbGaP: www.ncbi.nlm.nih.gov/projects/gap/cgi-bin/study.cgi? study_id=phs001215.v2.p2.
\end{abstract}

Keywords Cognitive function · Cognitive impairment · Genetic correlation · Genetic overlap · Type 2 diabetes

Electronic supplementary material The online version of this article (https://doi.org/10.1007/s00125-020-05101-y) contains peer-reviewed but undited supplementary material, which is available to authorised users.

Josephine Mollon

josephine.mollon@childrens.harvard.edu

1 Department of Psychiatry, Boston Children's Hospital, Harvard Medical School, 1 Autumn Street, BCH 3428, Boston, MA 02115 , USA

2 Department of Human Genetics, School of Medicine, University of Texas Rio Grande Valley, Brownsville, TX, USA

3 South Texas Diabetes and Obesity Institute, School of Medicine, University of Texas Rio Grande Valley, Brownsville, TX, USA
4 Olin Neuropsychiatry Research Center, Institute of Living, Hartford, CT, USA

5 Research Imaging Institute, University of Texas Health Science Center at San Antonio, San Antonio, TX, USA

6 Department of Psychiatry, University of Texas Health Science Center at San Antonio, San Antonio, TX, USA

7 Department of Genetics, Perelman School of Medicine, University of Pennsylvania, Philadelphia, PA, USA 


\section{Research in context}

What is already known about this subject?

- Type 2 diabetes has been associated with cognitive impairments

- Evidence suggests that these impairments are not sequelae of the illness

- Common genetic factors may influence both type 2 diabetes risk and cognitive dysfunction

\section{What is the key question?}

- Do the same genetic factors that influence type 2 diabetes risk also influence poor cognition?

\section{What are the new findings?}

- Negative genetic correlations between type 2 diabetes and performance on measures of attention, working memory and face memory suggest genetic overlap

- Cognitive performance was lowest in individuals with type 2 diabetes, highest in unaffected/unrelated individuals and intermediate in those related to an individual with type 2 diabetes

How might this impact on clinical practice in the foreseeable future?

- Cognitive impairment may be a useful endophenotype of type 2 diabetes and could help elucidate the pathophysiological underpinnings of this disease, eventually leading to improved detection and treatment

$\begin{array}{ll}\text { Abbreviations } \\ \text { CPT d' } & \text { Continuous Performance Test } \\ \text { CVLT } & \text { California Verbal Learning Test } \\ \text { ERV } & \text { Endophenotype ranking values } \\ \text { GOBS } & \text { Genetics of Brain Structure and Function } \\ \text { LDSC } & \text { Linkage disequilibrium score regression } \\ \text { mERV } & \text { Mean-based endophenotype ranking values } \\ \text { MR } & \text { Mendelian randomisation } \\ \text { PFMT } & \text { Penn Face Memory Test } \\ \text { PRS } & \text { Polygenic risk score } \\ \text { SOLAR } & \text { Sequential Oligogenetic Linkage Analysis } \\ & \text { Routines }\end{array}$

\section{Introduction}

Over 29 million Americans have diabetes and 90-95\% of these have type 2 diabetes [1]. If current trends continue, as many as one in three Americans are predicted to have diabetes by $2050[1,2]$, prompting Zimmet and colleagues to claim that type 2 diabetes is an 'epidemic' with profound societal consequences [3].

From the prospective of pathophysiology, the sevenfold increase in type 2 diabetes prevalence over the past 60 years [2] must be due to environmental factors (or the interaction of environmental factors with genetic background) since genetic variation on such a short timescale is relatively constant. Nevertheless, high concordance rates for type 2 diabetes in identical twins [4,5] and aggregation of type 2 diabetes in families $[6,7]$ suggest that genetic factors play an important role in illness liability. However, despite recent progress in delineating the genetic architecture of type 2 diabetes [8], only around $10 \%$ of the risk attributable to genetic factors has been identified [9]. A potential reason for the slow progress in demarcating genomic regions that confer type 2 diabetes risk is that the genetic architecture of the illness is highly heterogeneous [10]. One strategy to reduce this heterogeneity is the application of allied phenotypes or endophenotypes [11], defined as traits that are genetically related to, but not a symptom of, an illness. The endophenotype must show shared genetic aetiology with illness risk, such that the biological mechanisms underlying the endophenotype overlap with those that are disrupted in the disease [12]. Yet, despite the potential utility of the endophenotype strategy, relatively few studies have attempted to identify potential endophenotypes for type 2 diabetes.

While there are many potential medical complications of type 2 diabetes, cognitive impairment and dementia are increasingly recognised as clinically important [13]. Indeed, individuals with type 2 diabetes have a 1.5 times increased risk for Alzheimer's disease and other dementias [14], with longitudinal studies consistently reporting that type 2 diabetes in midlife is associated with increased risk of dementia in later life [14]. A recent meta-analysis of 2.3 million individuals, including more than 100,000 with dementia, found a $60 \%$ increased risk of any dementia in men and women with type 2 diabetes (women, pooled relative risk: 1.62; men, pooled relative risk: 1.58) [15]. Moreover, individuals with type 2 
diabetes have modest, yet reliable, cognitive decrements when compared with individuals without type 2 diabetes [16, 17]. For example, meta-analyses report small to moderate impairments on measures of processing speed (Cohen's $d-0.43$ to $-0.22[18-20])$, verbal declarative memory $(d=-0.51$ to $-0.27[18,20])$, visual declarative memory $(d=-0.26[18$, $20])$, executive functioning $(d=-0.52$ to $-0.25[18-20])$ and motor functioning $(d=-0.36$ [18]) in individuals with type 2 diabetes. Cognitive impairments are also present in individuals with recent-onset type 2 diabetes [21], adolescents who later develop diabetes [22] and in individuals with impaired glucose tolerance [23]. Thus, at least part of the cognitive impairment associated with type 2 diabetes appears to precede onset and may be related to risk for the illness.

Unlike other common sequelae of type 2 diabetes (e.g. retinopathy or peripheral neuropathy), cognitive impairments are only weakly associated with peripheral blood glucose levels or glucose regulation [24], suggesting that these impairments are not entirely due to current metabolic dysfunction (e.g. insulin resistance). Poor cognitive functioning also appears to be a risk factor for metabolic dysregulation [13], such as severe hypoglycaemic episodes [25], suggesting a bidirectional association between cognition and type 2 diabetes. Moreover, in a systematic review and synthesis of the literature, Biessels and colleagues [25] noted that effect sizes of cognitive impairment in type 2 diabetes are consistent across the lifespan and similar to those reported in individuals with impaired glucose tolerance [23], suggesting minimal influence of illness duration and/or age. Given evidence that cognitive impairments show relatively little association with clinical state, exist prior to illness onset and show minimal progression [13, 14], it is possible that at least some of the cognitive complications of type 2 diabetes reflect subtle biological changes associated with liability for type 2 diabetes. In other words, cognitive impairment may be an endophenotype of type 2 diabetes.

Using data from a large sample of Mexican-American individuals from extended pedigrees, we sought to find evidence for possible pleiotropy between cognitive functioning and type 2 diabetes, such that the genetic factors influencing these two traits overlap. Specifically, our aims were to: (1) estimate the heritability of type 2 diabetes and cognitive functioning in this sample; (2) quantify the genetic correlation between these two traits; and (3) test for the effect of duration of type 2 diabetes on cognitive functioning.

\section{Methods}

\section{Sample}

Participants were from the Genetics of Brain Structure and Function (GOBS) study [26, 27],which is part of the San
Antonio Family Heart Study (SAFH). Cognitive data and data on type 2 diabetes status were available for 1892 participants from 96 pedigrees (average [mean] family size, 19.2; range, 2-189). The sample was $60.4 \%$ female and had a mean age of 49.9 years (SD, 15.6; range, 18-97). GOBS data collection occurred between 2006 and 2016. Of the 1892 individuals, 402 received a type 2 diabetes diagnosis (see below), 1247 were related to an affected individual and 243 were unrelated to an affected individual (Table 1).

All participants were randomly selected from the community with the constraints that they were of Mexican-American ancestry, part of a large family, and lived in the San Antonio (TX, USA) region. All participants provided written informed consent. The institutional review board (IRB) at the University of Texas Science Center at San Antonio approved the study.

\section{Neurocognitive assessment}

Participants completed a 90 min neuropsychological test battery consisting of standard and computerised measures [28], including measures of attention, executive processing, working memory, declarative memory, language processing, intelligence and emotional processing. The vocabulary and matrix reasoning subtests of the Wechsler Abbreviated Scale of Intelligence (WASI) [29] provided an estimate of intelligence quotient (IQ). Participants were tested in their choice of language; 132 (7\%) participants were tested in Spanish and the remainder were tested in English.

\section{Type 2 diabetes diagnosis}

Participants were classified as having type 2 diabetes if they had a fasting glucose concentration $\geq 7.0 \mathrm{mmol} / \mathrm{l}$ and/or a $2 \mathrm{~h}$ glucose level $\geq 11.1 \mathrm{mmol} / 1$ after OGTT. Participants who did not meet these criteria, but reported current treatment with oral glucose-lowering agents or insulin, and a history of diabetes, were also classified as having type 2 diabetes.

Table 1 Basic demographics of the sample by degree of relatedness to an individual with type 2 diabetes

\begin{tabular}{lrll}
\hline Degree of relatedness & \multicolumn{1}{l}{$n$} & Age, mean (SD) & Sex, \% female \\
\hline Affected & 402 & $54.8(12.6)$ & 59.6 \\
First degree & 561 & $41.2(13.4)$ & 63.3 \\
Second degree & 337 & $34.3(14.9)$ & 57.0 \\
Third degree & 222 & $35.9(12.7)$ & 57.7 \\
Fourth degree & 105 & $29.4(9.6)$ & 61.0 \\
Fifth degree & 21 & $24.4(8.3)$ & 47.6 \\
Sixth degree & 1 & 19 & 100.0 \\
Unrelated & 243 & $45.5(15.2)$ & 63.0 \\
\hline
\end{tabular}




\section{Quantitative genetic analysis}

Univariate models All genetic analyses were performed using the Sequential Oligogenetic Linkage Analysis Routines (SOLAR) software [30]. SOLAR implements a maximum likelihood variance decomposition to determine the proportion of variation in a phenotype due to genetic and environmental influences by modelling the covariance amongst family members as a function of genetic proximity. This approach can handle pedigrees of arbitrary size and complexity and, thus, is optimally efficient with regard to extracting maximal genetic information. The simplest such decomposition is one where the additive genetic contribution of a trait is indexed by the heritability $\left(h^{2}\right)$. All cognitive measures and type 2 diabetes underwent univariate decomposition analysis to ensure they were significantly heritable. Raw continuous traits were subjected to rank-based inverse-normal transformation to ensure that they were normally distributed. Residualised traits were then generated by entering age, $\operatorname{age}^{2}$ and sex, and their interactions, as well as testing language and years of education, as fixed-effect covariates in all models. These residualised traits were used in all subsequent analyses. To control for multiple testing, the false discovery rate (FDR) was set at 5\% in all genetic and statistical models [31].

Bivariate models Bivariate polygenic models were used to decompose the phenotypic covariance between each neurocognitive measure and type 2 diabetes status into genetic and environmental constituents to determine the extent by which they were influenced by shared genetic effects. Specifically, bivariate polygenic analyses were performed to estimate phenotypic $\left(\rho_{p}\right)$, genetic $\left(\rho_{g}\right)$ and environmental $\left(\rho_{e}\right)$ correlations using the following equation: $\rho_{p}=\rho_{g} \sqrt{ }\left(h^{2} h^{2}{ }_{\mathrm{i}}\right)+$ $\rho_{e} \sqrt{ }\left[\left(1-h^{2}{ }_{\mathrm{e}}\right)\left(1-h^{2}{ }_{\mathrm{i}}\right)\right]$, where $h^{2}$ is the heritability of the endophenotype and $h^{2}$ is the heritability of the illness. The significance of these correlations was tested by comparing the $\log$ likelihood for two restricted models (with $\rho_{p}, \rho_{g}$ or $\rho_{e}$ constrained to equal 0 ) against the log likelihood for the model in which these parameters were estimated. A significant phenotypic correlation is evidence for a phenotypic association (i.e. including both genetic and environmental influences) between neurocognitive measures and a type 2 diabetes diagnosis. A significant environmental correlation is evidence for a non-genetic factor jointly influencing both traits. A significant genetic correlation is evidence for pleiotropy suggesting that a gene or set of genes jointly influences both phenotypes. It is worth noting that there are multiple possible interpretations of genetic correlations. While the same genetic variants may contribute both to type 2 diabetes risk and cognitive functioning (horizontal pleiotropy), genetic variants related to type 2 diabetes risk may also have indirect effects on cognition (vertical pleiotropy) [32]. Nevertheless, the mechanisms underlying the observed genetic correlations between type 2 diabetes and cognitive impairment do not deter from the potential use of cognitive functioning as an endophenotype for type 2 diabetes. We also used bivariate models to decompose the phenotypic covariance between each neurocognitive measure and BMI, as well as between each neurocognitive measure and waist circumference.

Endophenotype Ranking Values Parameters from these bivariate models were used to calculate endophenotype ranking values (ERVs). The ERV objectively prioritises potential endophenotypes for use in molecular genetics analyses [33]. The ERV represents the standardised genetic covariance between an endophenotype and an illness, defined as: ERV $=\sqrt{ }\left(h^{2}{ }_{\mathrm{e}} h^{2}{ }_{\mathrm{i}}\right)\left|\rho_{g}\right|$, where $h_{\mathrm{e}}^{2}$ is the heritability of the endophenotype, $h^{2}{ }_{\mathrm{i}}$ is the heritability of the illness and $\rho_{g}$ is their genetic correlation. The ERV provides a measure between 0 and 1, with higher values indicating a stronger combination of genetic signal and relationship to disease.

Mean-based ERV calculation The mean-based ERV (mERV) is an extension of the ERV. For details on the derivation of the mERV, see Glahn et al [34]. Briefly, the mERV leverages the many coefficients of relationship that exist in extendedpedigree data. The coefficient of relationship refers to the average (mean) number of alleles held in common between individuals. For example, first-degree relatives (e.g. full siblings or parents) share, on average, $50 \%$ of their alleles, whilst second-degree relatives (e.g. grandparents or aunts/ uncles) share $25 \%$, third-degree relatives (e.g. greatgrandparents or great aunts/uncles) share $12.5 \%$ and so on. Thus, it is possible, given an individual with a disease, to index all other pedigree members by their degree of relatedness to that individual. For non-affected individuals with more than one relative with type 2 diabetes, the highest degree of relatedness is used. This scalar can then be used to perform a fixed-effect single-degree-of-freedom test within the univariate variance components analysis outlined above, providing an estimate of the standardised genetic covariance between the potential endophenotype and illness risk. The mERV can then be used in the same way as the ERV to rank potential endophenotypes by their degree of standardised genetic overlap with illness risk. In the present paper, the mERV was applied to type 2 diabetes and all neurocognitive measures with statistically significant genetic correlations.

\section{Statistical analyses}

We used ANOVA models, implemented in the statistical programming language $\mathrm{R}$ [35], to test for the effect of duration of type 2 diabetes on neurocognitive functioning. Participants with type 2 diabetes were categorised into two illness duration groups: (1) duration of less than 10 years; and (2) duration of 10 or more years. Neurocognitive scores were residualised in 
SOLAR for sex and testing language, then subsequently for sex, testing language and age. Finally, the three groups (unaffected, duration $<10$ years, duration $\geq 10$ years) were matched by age using the 'MatchIt' package in R.

We also examined the effect of duration of type 2 diabetes on neurocognitive functioning using linear regression models. Duration in years was modelled onto cognitive functioning, both as linear and quadratic functions, the latter to account for potential nonlinearity in the association between type 2 diabetes duration and cognitive functioning.

\section{Results}

\section{Evidence for pleiotropy between type 2 diabetes and neurocognition}

Table 2 shows results of univariate and bivariate genetic analyses of type 2 diabetes on neurocognitive functioning. All neurocognitive measures were significantly heritable $\left(h^{2}\right.$ range, $0.17-0.59)$, as was type 2 diabetes $(h=0.59 ; p=6 \times$ $\left.10^{-14}\right)$. Significant phenotypic correlations were observed between type 2 diabetes and measures of attention (Continuous Performance Test [CPT d']: $\rho_{p}=-0.143, p=$ 0.001), verbal memory (California Verbal Learning Test
[CVLT] recall: $\left.\rho_{p}=-0.111, p=0.004\right)$, and face memory (Penn Face Memory Test [PFMT]: $\rho_{p}=-0.127, p=0.002$; PFMT Delayed: $\rho_{p}=-0.148, p=2 \times 10^{-4}$ ). These statistically significant phenotypic correlations were in line with standardised mean difference effect sizes (Fig. 1). Significant genetic correlations were observed between type 2 diabetes and CPT d' $\left(\rho_{g}=-0.401, p=0.001\right)$, digit span backward $\left(\rho_{g}=-0.380, p=0.005\right), \operatorname{PFMT}\left(\rho_{g}=-0.476, p=2 \times 10^{-4}\right)$ and PFMT Delayed $\left(\rho_{g}=-0.376, p=0.005\right)$, suggesting overlap between the genetic factors influencing type 2 diabetes and performance on measures of attention, working memory and face memory, respectively.

Figure 2 shows results of mERV analyses. Standardised genetic covariances were statistically significant for all cognitive measures: CPT d' $(\beta=-0.219, p=0.005)$, digit span backward $(\beta=-0.326, p=0.035)$, PFMT $(\beta=-0.171, p=$ $0.023)$ and PFMT Delayed $(\beta=-0.215, p=0.005)$. However, the effect of relatedness on cognition differed between these measures; for CPT d', individuals with type 2 diabetes had the lowest scores, followed by their first-degree relatives and then their second- to sixth- degree relatives, while unaffected/unrelated individuals scored the highest. For the digit span backward, individuals with type 2 diabetes and their first-degree relatives had the lowest scores, followed by their second- to sixth- degree relatives, and unaffected/

Table 2 Results of genetic analyses of diabetes and cognitive measures

\begin{tabular}{|c|c|c|c|c|c|c|c|c|c|}
\hline \multirow[b]{2}{*}{ Cognitive measure } & \multicolumn{2}{|c|}{ Heritability } & \multicolumn{2}{|c|}{ Phenotypic correlation } & \multicolumn{2}{|c|}{ Environmental correlation } & \multicolumn{2}{|c|}{ Genetic correlation } & \multirow[b]{2}{*}{ ERV } \\
\hline & $h^{2}$ & $p$ & $\rho_{p}$ & $p$ & $\rho_{e}$ & $p$ & $\rho_{g}$ & $p$ & \\
\hline Semantic fluency & 0.219 & $1 \times 10^{-7 *}$ & -0.043 & 0.277 & 0.022 & 0.821 & -0.153 & 0.310 & 0.141 \\
\hline Verbal fluency & 0.390 & $1 \times 10^{-18 *}$ & -0.045 & 0.254 & 0.056 & 0.609 & -0.152 & 0.219 & 0.187 \\
\hline Digit symbol substitution & 0.320 & $5 \times 10^{-13 *}$ & -0.018 & 0.668 & -0.144 & 0.167 & 0.127 & 0.333 & 0.158 \\
\hline Trail-Making A & 0.167 & $2 \times 10^{-5 *}$ & 0.023 & 0.561 & 0.027 & 0.778 & 0.024 & 0.888 & 0.048 \\
\hline CPT d' & 0.380 & $1 \times 10^{-17} *$ & -0.143 & $0.001 *$ & 0.087 & 0.402 & -0.401 & $0.001 *$ & 0.296 \\
\hline Digit span forward & 0.424 & $2 \times 10^{-22} *$ & -0.055 & 0.170 & 0.007 & 0.949 & -0.115 & 0.327 & 0.171 \\
\hline Digit span backward & 0.307 & $1 \times 10^{-13} *$ & -0.044 & 0.275 & 0.223 & 0.029 & -0.380 & $0.005^{*}$ & 0.262 \\
\hline Letter number sequencing & 0.272 & $8 \times 10^{-12} *$ & -0.045 & 0.259 & 0.066 & 0.496 & -0.203 & 0.133 & 0.180 \\
\hline PCET correct & 0.241 & $4 \times 10^{-10} *$ & -0.076 & 0.047 & -0.072 & 0.447 & -0.095 & 0.501 & 0.117 \\
\hline Spatial working memory & 0.272 & $2 \times 10^{-12 *}$ & -0.059 & 0.122 & 0.027 & 0.773 & -0.186 & 0.167 & 0.172 \\
\hline Trail-Making B & 0.305 & $2 \times 10^{-11} *$ & 0.052 & 0.195 & 0.030 & 0.765 & 0.085 & 0.530 & 0.124 \\
\hline CVLT learning & 0.373 & $6 \times 10^{-16 *}$ & -0.086 & 0.027 & 0.043 & 0.678 & -0.229 & 0.070 & 0.224 \\
\hline CVLT recall & 0.355 & $1 \times 10^{-16 *}$ & -0.111 & $0.004 *$ & -0.062 & 0.529 & -0.173 & 0.160 & 0.191 \\
\hline PFMT & 0.337 & $3 \times 10^{-18 *}$ & -0.127 & $0.002 *$ & 0.159 & 0.108 & -0.476 & $2 \times 10^{-4 *}$ & 0.305 \\
\hline PFMT Delayed & 0.354 & $2 \times 10^{-16 *}$ & -0.148 & $2 \times 10^{-4 *}$ & 0.034 & 0.742 & -0.376 & $0.005^{*}$ & 0.270 \\
\hline Emotion recognition & 0.212 & $1 \times 10^{-7} *$ & -0.058 & 0.130 & 0.103 & 0.271 & -0.341 & 0.029 & 0.202 \\
\hline Matrix reasoning & 0.334 & $1 \times 10^{-17 *}$ & -0.058 & 0.142 & -0.031 & 0.751 & -0.095 & 0.448 & 0.137 \\
\hline Vocabulary & 0.585 & $8 \times 10^{-34 *}$ & -0.053 & 0.187 & 0.064 & 0.605 & -0.137 & 0.209 & 0.216 \\
\hline IQ & 0.576 & $5 \times 10^{-35 *}$ & -0.049 & 0.228 & 0.030 & 0.810 & -0.106 & 0.327 & 0.189 \\
\hline
\end{tabular}

*Significant after correction for multiple testing $(\mathrm{FDR}=0.05)$

IQ, intelligence quotient; PCET, Penn Conditional Exclusion Test 


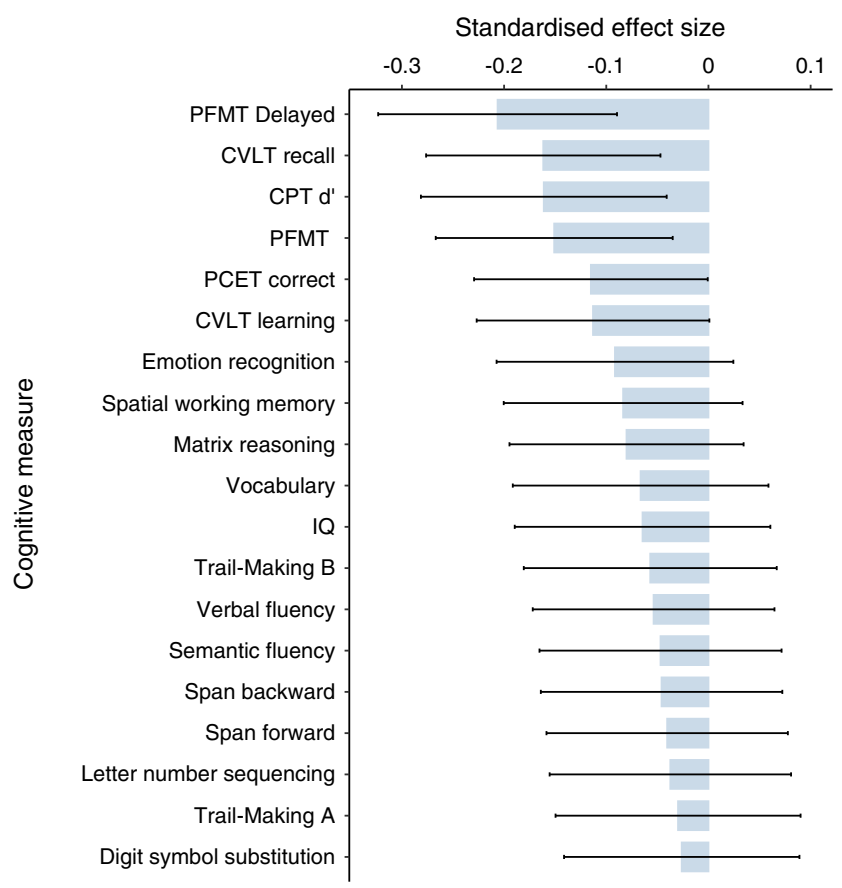

Fig. 1 Standardised effect sizes and 95\% CIs for all residualised cognitive measures comparing individuals with type 2 diabetes with individuals without (control data from unaffected individuals set at 0 for all measures). IQ, intelligence quotient; PCET, Penn Conditional Exclusion Test

unrelated individuals scored the highest. For PFMT and PFMT Delayed, individuals with type 2 diabetes had the lowest scores, unaffected/unrelated individuals had the
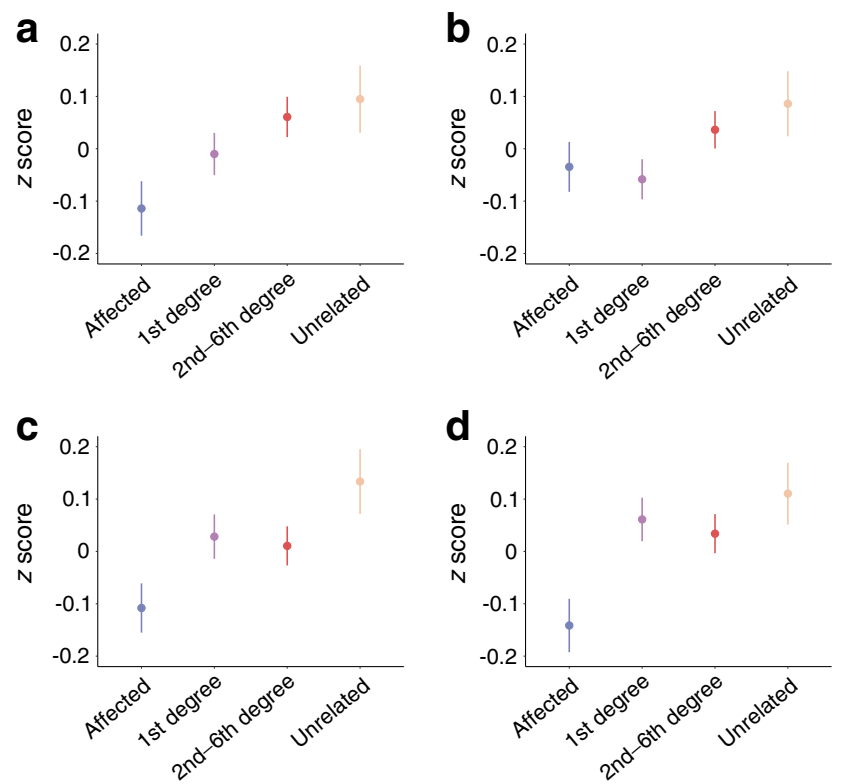

Fig. 2 mERV analysis of cognitive measures with statistically significant genetic correlations, plotted by degree of relatedness. (a) CPT $\mathrm{d}^{\prime}: \beta=$ $-0.219, p=0.005$; (b) digit span backward: $\beta=-0.326, p=0.035$; (c) PFMT: $\beta=-0.171, p=0.023$; (d) PFMT Delayed: $\beta=-0.215, p=0.005$ highest scores, and those related to an individual with type 2 diabetes had intermediate scores.

\section{No evidence for pleiotropy between BMI or waist circumference and neurocognition}

Bivariate genetic analyses of BMI and waist circumference on cognitive functioning are shown in the electronic supplementary material (ESM) Table 1 and Table 2, respectively. Phenotypic, environmental and genetic correlations did not reach significance for any neurocognitive measure after correction for multiple testing, except for the phenotypic correlation between CPT $\mathrm{d}^{\prime}$ and waist circumference $\left(\rho_{p}=\right.$ -0.084 ; ESM Table 2).

\section{Deleterious effects of illness duration on neurocognition are confounded by age}

Demographic characteristics of the unmatched and matched samples grouped by duration of type 2 diabetes (unaffected, duration $<10$ years, duration $\geq 10$ years) are shown in ESM Table 3. ESM Fig. 1 shows age distributions for the agematched groups. In the unmatched sample, the groups separated by type 2 diabetes duration differed significantly by age $(p<0.001)$; in the matched sample, there was no statistically significant difference in age ( $p=0.714$; ESM Table 3$)$.

Analyses of illness duration group status on neurocognitive functioning are shown in Fig. 3 and ESM Table 4. When adjusting for sex, the duration $<10$ years group had lower cognitive scores than the unaffected individuals for 13 out of the 19 cognitive measures, while the duration $\geq 10$ years group showed lower scores than the unaffected individuals for 16 out of the 19 measures (ESM Table 4). Moreover, the duration $\geq 10$ years group showed lower scores than the duration $<10$ years group for digit symbol substitution $(\beta=-0.24$, $p=0.017)$, Trail-Making A $(\beta=-0.36, p=0.001)$, digit span forward $(\beta=-0.32, p=0.003)$, Trail-Making $\mathrm{B}(\beta=-0.24$, $p=0.041)$, CVLT recall $(\beta=-0.26, p=0.018)$ and emotion recognition $(\beta=-0.28, p=0.013)$. Matching the groups by age attenuated most of these group differences, with the duration $<10$ years group performing worse than the unaffected individuals for CPT $\mathrm{d}^{\prime}(\beta=-0.30, p=0.006)$, PFMT $(\beta=$ $-0.27, p=0.015)$ and PFMT Delayed $(\beta=-0.27, p=0.014)$, the duration $\geq 10$ years group performing worse than the unaffected individuals for CPT $\mathrm{d}^{\prime}(\beta=-0.26, p=0.026)$, digit span forward $(\beta=-0.23 p=0.047)$, CVLT recall $(\beta=-0.23, p=$ $0.049)$ and PFMT Delayed $(\beta=-0.23, p=0.049)$, and the duration $\geq 10$ years group performing worse than the duration $<10$ years group for digit span forward $(\beta=-0.34, p=0.010)$. Adjusting additionally for age further attenuated these group differences, with the duration $<10$ years group performing worse than the unaffected individuals for PFMT $(\beta=-0.18$, $p=0.042)$ and PFMT Delayed $(\beta=-0.22, p=0.009)$, the 


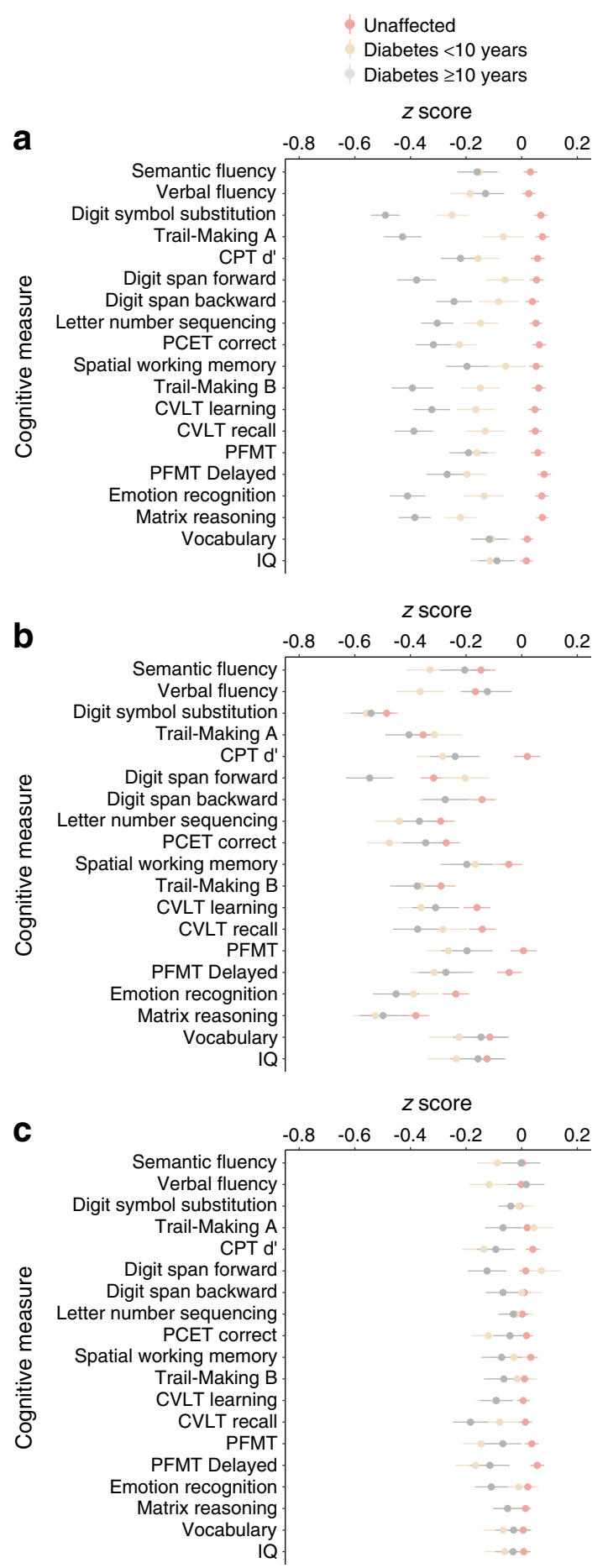

Fig. 3 Means and SE of cognitive measures by illness duration, (a) adjusting for sex, (b) adjusting for sex and matched for age or (c) adjusting for sex, age, age ${ }^{2}$, age $\times$ sex, age ${ }^{2} \times$ sex. IQ, intelligence quotient; PCET, Penn Conditional Exclusion Test

duration $\geq 10$ years group performing worse than the unaffected individuals for CVLT recall $(\beta=-0.20, p=0.011)$, and no statistically significant differences between the duration $\geq 10$ years and duration $<10$ years groups.
Analyses of illness duration on cognitive functioning, with illness duration years as linear and quadratic functions, are shown in ESM Table 5 and ESM Table 6, respectively. Results were similar to above, with a significant effect of duration (linear function) on digit symbol substitution $(\beta=$ $-0.019, p=0.001)$, Trail-Making A $(\beta=-0.023, p=0.002)$, digit span forward $(\beta=-0.016, p=0.021)$, digit span backward $(\beta=-0.013, p=0.046)$, letter number sequencing $(\beta=$ $-0.014, p=0.021)$, Trail-Making B $(\beta=-0.021, p=0.006)$, CVLT learning $(\beta=-0.014, p=0.023)$, CVLT recall $(\beta=$ $-0.014, p=0.031)$ and emotion recognition $(\beta=-0.015, p=$ 0.021) (ESM Table 5 and ESM Fig. 2). A significant effect of duration as a quadratic function, was also seen on digit symbol substitution $(\beta=-0.0006, p=0.001)$, Trail-Making $\mathrm{A}(\beta=$ $-0.0007, p=0.014)$, digit span forward $(\beta=-0.0005, p=$ $0.034)$, letter number sequencing $(\beta=-0.0005, p=0.011)$, Trail-Making B $(\beta=-0.0007, p=0.011)$, CVLT learning $(\beta=-0.0005, p=0.014)$, and emotion recognition $(\beta=$ $-0.0005, p=0.043$ ) (ESM Table 6 and ESM Fig. 3). No statistically significant effect of illness duration was seen when groups were matched for age, except for digit span forward $(\beta=-0.019, p=0.009$ for effect of duration as a linear function [ESM Table 5]; $\beta=-0.0005, p=0.032$ for effect of duration as a quadratic function [ESM Table 6]). There were no statistically significant effects of illness duration after further adjustment for age.

\section{Discussion}

Using a large sample of Mexican-American individuals from extended pedigrees, we established evidence for pleiotropy between cognitive impairment and type 2 diabetes. Significant genetic correlations were observed between type 2 diabetes and measures of attention, working memory and face memory, suggesting genetic overlap between type 2 diabetes and these cognitive domains. Moreover, significant genetic correlations were not observed between either BMI or waist circumference and cognitive performance, suggesting that the genetic overlap between type 2 diabetes and cognitive functioning is specific to the illness and not seen with general obesity factors. Finally, although there was an effect of duration of type 2 diabetes on magnitude of cognitive impairment, with individuals with a longer duration of illness showing larger impairments than individuals with a shorter duration of illness, these group differences were confounded by age. Our findings add to current knowledge about the pathophysiology of type 2 diabetes in several important ways.

First, the finding of pleiotropy between cognitive functioning and type 2 diabetes may be an important step forward in delineating the genetic underpinnings of type 2 diabetes, which affects an exponentially increasing number of individuals worldwide. While there has been progress in delineating 
the genetic architecture of type 2 diabetes [8], some argue that the illness remains 'a geneticist's nightmare' [9]. One strategy for identifying risk genes for type 2 diabetes is the application of endophenotypes $[11,36]$, i.e. traits that are genetically related to the illness. However, while there is strong evidence to suggest that individuals with type 2 diabetes show cognitive impairments, few studies have sought to establish whether these impairments are genetically correlated with the illness. Our finding of genetic overlap between type 2 diabetes and measures of memory, working memory and attention is in line with evidence that the most consistent impairments in individuals with type 2 diabetes are in the domains of memory and executive function [18-20]. Moreover, this finding provides evidence for one of the principle criteria of endophenotypes: that the same genes that convey risk for the illness also influence the endophenotype [36]. Similarly, analyses of data from the UK Biobank and 24 international genome-wide association studies (GWAS) consortia showed that higher polygenic risk for type 2 diabetes was associated with decreased likelihood of obtaining a college degree [37]. However, a higher polygenic risk score (PRS) for type 2 diabetes was not associated with verbal reasoning, reaction time or memory in this sample [37], and no significant genetic correlations were reported between type 2 diabetes and any of the cognitive or education phenotypes when using linkage disequilibrium score regression (LDSC) [37]. Moreover, Mendelian randomisation (MR) analyses in the same sample provided no evidence for a causal association between type 2 diabetes and cognitive ability or educational attainment [38]. However, the methods used in the UK Biobank (PRS, LDSC and MR) primarily capture common genetic variance, while the genetic correlations observed in our study may be driven, at least in part, by rare genetic variants. In an ageing cohort, genetic risk of type 2 diabetes was positively associated with fluid intelligence, but no association was detected between type 2 diabetes PRS and verbal intelligence, memory or processing speed [39]. Thus, while our findings suggest that cognitive impairment may be a useful endophenotype of type 2 diabetes, future studies are needed to disentangle the genetic overlap between these traits at different ages, as well as across different cognitive domains. It is also worth noting that there are multiple possible interpretations of genetic correlations. While the same genetic variants may contribute both to type 2 diabetes risk and cognitive functioning, genetic variants related to type 2 diabetes risk may also have indirect effects on cognition, and genetic variants related to cognition may even have indirect effects on type 2 diabetes [32]. Nevertheless, the mechanisms underlying the observed genetic correlations between type 2 diabetes and cognitive impairment do not deter from the potential utility of cognitive functioning as an endophenotype for type 2 diabetes.

Second, we did not find evidence for a genetic association between cognitive function and either BMI or waist circumference. Previous evidence from twin and molecular genetic models indicate inconsistent findings regarding the genetic association between BMI and cognitive functioning, with reports of medium [40], small [41] and null [42] genetic correlations between the two traits. Even at the phenotypic level, the association between BMI and cognitive functioning is unclear, with reports of no association [43], cognitive impairment $[44,45]$ and even improved cognitive performance $[46,47]$ with higher BMI. We found null to small phenotypic and genetic correlations between neurocognition and both BMI and waist circumference, and none of these reached statistical significance after correction for multiple testing. Thus, any association between obesity indices and cognitive impairment may be due to environmental, rather than genetic, risk factors. Alternatively, genetic risk factors may interact with environmental changes throughout the life course, such that obesity-related pathology leading to cognitive impairment and/or decline may develop gradually over the course of many years [48]. Future studies are needed to determine whether this potential association between obesity indices and cognitive performance is moderated by age.

Finally, we found greater cognitive impairment in individuals with a longer duration of type 2 diabetes, but also that this group difference was attenuated when adjusting for age. Since age increases with duration of type 2 diabetes, adjusting for age undoubtedly attenuates part of the effect of duration on cognitive functioning. Nevertheless, this finding is in line with evidence that the magnitude of cognitive impairment associated with type 2 diabetes remains relatively stable throughout the lifespan [25]. Similarly, cognitive impairments are already present in individuals with recent-onset type 2 diabetes [21] and even in adolescents who later develop diabetes [22]. Moreover, a negligible effect of illness duration on cognition aligns with the finding of genetic overlap between type 2 diabetes and cognitive impairment, as well as the notion of a bidirectional relationship between these traits [13]. However, there have also been reports of an association between duration of type 2 diabetes and magnitude of cognitive dysfunction $[49,50]$. Future longitudinal studies, which include individuals throughout premorbid and post-onset stages of type 2 diabetes, as well as repeating cognitive assessments, are needed to fully disentangle the complex mechanisms underlying the relationship between type 2 diabetes and poor cognitive outcomes. Moreover, future studies that use additional measures, such as blood glucose level and family history of type 2 diabetes, to examine whether some portion of the cognitive impairment associated with type 2 diabetes arises as a consequence of the illness, may also help elucidate these mechanisms.

This study has some limitations. First, due to the crosssectional nature of this study, it is not possible to draw inferences about timing. While we found evidence for pleiotropy between type 2 diabetes and cognitive 
impairment, it remains unclear how these overlapping genetic factors might interact with other genetic and environmental risk factors over the lifecourse. Future longitudinal studies will help elucidate the complex mechanisms underlying risk for both type 2 diabetes and cognitive impairment, as well as potential developmental periods for optimal intervention and prevention. Second, the aim of this study was to examine pleiotropy between type 2 diabetes and cognitive impairment, but other potential explanations for the association between type 2 diabetes and cognitive impairment warrant further examination. As outlined above, future studies that are able to examine whether some portion of the cognitive impairment associated with type 2 diabetes arises due to the illness, or even whether some portion of type 2 diabetes risk is consequential to poor cognitive functioning, may yield interesting results.

Using a large sample of Mexican-American individuals from extended pedigrees, we established evidence for pleiotropy between impairment on measures of attention, working memory and memory, and type 2 diabetes. Thus, cognitive impairment may be a useful endophenotype of type 2 diabetes and may help elucidate the pathophysiological underpinnings of this chronic illness, which affects an large number of individuals worldwide. Future longitudinal studies will help disentangle these pathophysiological mechanisms over the life course in order to inform treatment strategies and intervention efforts.

Acknowledgements We are very grateful to all the participants of the GOBS study. We thank M. Woolsey (Research Imaging Institute, University of Texas Health Science Center at San Antonio, TX, USA) for her role in data collection.

Data availability The data analysed in this study is available in dbGaP: https://www.ncbi.nlm.nih.gov/projects/gap/cgi-bin/study.cgi?study_id= phs001215.v2.p2.

Funding Financial support for this study was provided by the National Institutes of Health grants MH059490 (principal investigator [PI]: JB), MH078143 (PI: DCG), MH078111 (PI: JB), MH083824 (PIs: DCG/JB) and AG058464 (PIs: DCG/JB). The sponsors had no role in the design and conduct of the study; collection, management, analysis and interpretation of the data; and preparation, review or approval of this manuscript.

Duality of interest The authors declare that there is no duality of interest associated with this manuscript.

Contribution statement JEC, JB and DCG designed and conceptualised the study. JM performed statistical analysis. DCG and JM wrote the first and successive drafts of the manuscript. All authors contributed to acquisition, analysis or interpretation of data, and drafting or critical revision of the manuscript for important intellectual content. All authors approved the final version of the paper to be published. JB and DCG obtained funding. DCG attests that all listed authors meet authorship criteria and that no others meeting the criteria have been omitted. JM and DCG are responsible for the integrity of the work as a whole.

\section{References}

1. Centers for Disease Control and Prevention (2014) National diabetes statistics report: estimates of diabetes and its burden in the United States. CDC, Atlanta

2. Menke A, Casagrande S, Geiss L, Cowie CC (2015) Prevalence of and trends in diabetes among adults in the United States, 19882012. JAMA 314(10):1021-1029. https://doi.org/10.1001/jama. 2015.10029

3. Zimmet P, Alberti K, Shaw J (2001) Global and societal implications of the diabetes epidemic. Nature 414(6865):782-787. https:// doi.org/10.1038/414782a

4. Kyvik KO, Green A, Beck-Nielsen H (1995) Concordance rates of insulin dependent diabetes mellitus: a population based study of young Danish twins. BMJ 311(7010):913-917. https://doi.org/10. 1136/bmj.311.7010.913

5. Willemsen G, Ward KJ, Bell CG et al (2015) The concordance and heritability of type 2 diabetes in 34,166 twin pairs from international twin registers: the discordant twin (DISCOTWIN) consortium. Twin Res Hum Genet 18(6):762-771. https://doi.org/10.1017/thg. 2015.83

6. Almgren P, Lehtovirta M, Isomaa B et al (2011) Heritability and familiality of type 2 diabetes and related quantitative traits in the Botnia Study. Diabetologia 54(11):2811-2819. https://doi.org/10. 1007/s00125-011-2267-5

7. Meigs JB, Cupples LA, Wilson PW (2000) Parental transmission of type 2 diabetes: the Framingham Offspring Study. Diabetes 49(12): 2201-2207. https://doi.org/10.2337/diabetes.49.12.2201

8. Fuchsberger C, Flannick J, Teslovich TM et al (2016) The genetic architecture of type 2 diabetes. Nature 536(7614):41-47. https:// doi.org/10.1038/nature18642

9. Rich SS (2016) Diabetes: still a geneticist's nightmare. Nature 536(7614):37-38. https://doi.org/10.1038/nature18906

10. McClellan J, King MC (2010) Genetic heterogeneity in human disease. Cell 141(2):210-217. https://doi.org/10.1016/j.cell.2010. 03.032

11. Gottesman II, Gould TD (2003) The endophenotype concept in psychiatry: etymology and strategic intentions. Am J Psychiatr 160(4):636-645. https://doi.org/10.1176/appi.ajp.160.4.636

12. Knowles EE, Meikle PJ, Huynh K et al (2017) Serum phosphatidylinositol as a biomarker for bipolar disorder liability. Bipolar Disord 19(2):107-115. https://doi.org/10.1111/bdi.12468

13. Stoeckel LE, Arvanitakis Z, Gandy S et al (2016) Complex mechanisms linking neurocognitive dysfunction to insulin resistance and other metabolic dysfunction. F1000Res 5:353. https://doi.org/10. 12688/f1000research.8300.2

14. Biessels GJ, Staekenborg S, Brunner E, Brayne C, Scheltens P (2006) Risk of dementia in diabetes mellitus: a systematic review. Lancet Neurol 5(1):64-74. https://doi.org/10.1016/S14744422(05)70284-2

15. Chatterjee S, Peters SA, Woodward M et al (2016) Type 2 diabetes as a risk factor for dementia in women compared with men: a pooled analysis of 2.3 million people comprising more than 100 , 000 cases of dementia. Diabetes Care 39(2):300-307. https://doi. org $110.2337 / \mathrm{dc} 15-1588$

16. van den Berg E, Kloppenborg RP, Kessels RP, Kappelle LJ, Biessels GJ (2009) Type 2 diabetes mellitus, hypertension, dyslipidemia and obesity: a systematic comparison of their impact on cognition. Biochim Biophys Acta 1792(5):470-481. https://doi. org/10.1016/j.bbadis.2008.09.004

17. Allen KV, Frier BM, Strachan MW (2004) The relationship between type 2 diabetes and cognitive dysfunction: longitudinal studies and their methodological limitations. Eur J Pharmacol 490(1-3):169-175. https://doi.org/10.1016/j.ejphar.2004.02.054 
18. Palta P, Schneider AL, Biessels GJ, Touradji P, Hill-Briggs F (2014) Magnitude of cognitive dysfunction in adults with type 2 diabetes: a meta-analysis of six cognitive domains and the most frequently reported neuropsychological tests within domains. J Int Neuropsychol Soc 20(3):278-291. https://doi.org/10.1017/ S1355617713001483

19. Vincent C, Hall PA (2015) Executive function in adults with type 2 diabetes: a meta-analytic review. Psychosom Med 77(6):631-642. https://doi.org/10.1097/PSY.0000000000000103

20. Monette MC, Baird A, Jackson DL (2014) A meta-analysis of cognitive functioning in nondemented adults with type 2 diabetes mellitus. Can J Diabetes 38(6):401-408. https://doi.org/10.1016/j. jcjd.2014.01.014

21. Ruis C, Biessels GJ, Gorter KJ, van den Donk M, Kappelle LJ, Rutten GE (2009) Cognition in the early stage of type 2 diabetes. Diabetes Care 32(7):1261-1265. https://doi.org/10.2337/dc082143

22. Ryan C, Vega A, Drash A (1985) Cognitive deficits in adolescents who developed diabetes early in life. Pediatrics 75(5):921-927

23. Yates KF, Sweat V, Yau PL, Turchiano MM, Convit A (2012) Impact of metabolic syndrome on cognition and brain: a selected review of the literature. Arterioscler Thromb Vasc Biol 32(9):2060 2067. https://doi.org/10.1161/ATVBAHA.112.252759

24. Geijselaers SL, Sep SJ, Stehouwer CD, Biessels GJ (2015) Glucose regulation, cognition, and brain MRI in type 2 diabetes: a systematic review. Lancet Diabetes Endocrinol 3(1):75-89. https://doi.org/ 10.1016/S2213-8587(14)70148-2

25. Biessels GJ, Strachan MW, Visseren FL, Kappelle LJ, Whitmer RA (2014) Dementia and cognitive decline in type 2 diabetes and prediabetic stages: towards targeted interventions. Lancet Diabetes Endocrinol 2(3):246-255. https://doi.org/10.1016/S2213-8587(13) 70088-3

26. Olvera R, Bearden C, Velligan D et al (2011) Common genetic influences on depression, alcohol, and substance use disorders in Mexican-American families. Am J Med Genet Part B 156(5):561568

27. McKay DR, Knowles EE, Winkler AA et al (2014) Influence of age, sex and genetic factors on the human brain. Brain Imaging Behav 8(2):143-152. https://doi.org/10.1007/s11682-013-9277-5

28. Glahn D, Almasy L, Barguil M et al (2010) Neurocognitive endophenotypes for bipolar disorder identified in multiplex multigenerational families. Arch Gen Psychiatry 67(2):168-177. https:// doi.org/10.1001/archgenpsychiatry.2009.184

29. Wechsler D (1999) Wechsler abbreviated scale of intelligence. The Psychological Corporation, San Antonio

30. Almasy L, Blangero J (1998) Multipoint quantitative-trait linkage analysis in general pedigrees. Am J Hum Genet 62(5):1198-1211. https://doi.org/10.1086/301844

31. Benjamini Y, Yekutieli DJTaos (2001) The control of the false discovery rate in multiple testing under dependency. 29(4): 11651188

32. Solovieff N, Cotsapas C, Lee PH, Purcell SM, Smoller JW (2013) Pleiotropy in complex traits: challenges and strategies. Nat Rev Genet 14(7):483-495. https://doi.org/10.1038/nrg3461

33. Glahn DC, Curran JE, Winkler AM et al (2012) High dimensional endophenotype ranking in the search for major depression risk genes. Biol Psychiatry 71(1):6-14. https://doi.org/10.1016/j. biopsych.2011.08.022

34. Glahn DC, Williams JT, McKay DR, et al (2015) Discovering schizophrenia endophenotypes in randomly ascertained pedigrees. 77(1): 75-83

35. R Development Core Team R (2011) R: a language and environment for statistical computing. R Foundation for Statistical Computing, Vienna
36. Glahn DC, Knowles EE, McKay DR et al (2014) Arguments for the sake of endophenotypes: examining common misconceptions about the use of endophenotypes in psychiatric genetics. Am J Med Genet Part B 165(2):122-130

37. Hagenaars SP, Harris SE, Davies G et al (2016) Shared genetic aetiology between cognitive functions and physical and mental health in UK Biobank $(\mathrm{N}=112$ 151) and 24 GWAS consortia. Mol Psychiatry 21(11):1624-1632. https://doi.org/10.1038/mp.2015. 225

38. Hagenaars SP, Gale CR, Deary IJ, Harris SE (2017) Cognitive ability and physical health: a Mendelian randomization study. Sci Rep 7(1):2651. https://doi.org/10.1038/s41598-017-02837-3

39. Luciano M, Mottus R, Harris SE et al (2014) Predicting cognitive ability in ageing cohorts using type 2 diabetes genetic risk. Diabet Med 31(6):714-720. https://doi.org/10.1111/dme.12389

40. Marioni RE, Yang J, Dykiert D et al (2016) Assessing the genetic overlap between BMI and cognitive function. Mol Psychiatry 21(10):1477-1482. https://doi.org/10.1038/mp.2015.205

41. Frazier-Wood AC, Carnell S, Pena O et al (2014) Cognitive performance and BMI in childhood: shared genetic influences between reaction time but not response inhibition. Obesity 22(11):23122318. https://doi.org/10.1002/oby.20862

42. Benyamin B, Wilson V, Whalley LJ, Visscher PM, Deary IJ (2005) Large, consistent estimates of the heritability of cognitive ability in two entire populations of 11-year-old twins from Scottish mental surveys of 1932 and 1947. Behav Genet 35(5):525-534. https://doi. org/10.1007/s10519-005-3556-x

43. Yesavage JA, Kinoshita LM, Noda A et al (2014) Effects of body mass index-related disorders on cognition: preliminary results. Diabetes Metab Syndr Obes 7:145-151. https://doi.org/10.2147/ DMSO.S60294

44. Sabia S, Kivimaki M, Shipley MJ, Marmot MG, Singh-Manoux A (2008) Body mass index over the adult life course and cognition in late midlife: the Whitehall II Cohort Study. Am J Clin Nutr 89(2): 601-607. https://doi.org/10.3945/ajcn.2008.26482

45. Cournot M, Marquie J, Ansiau D et al (2006) Relation between body mass index and cognitive function in healthy middle-aged men and women. Neurology 67(7):1208-1214. https://doi.org/10. 1212/01.wnl.0000238082.13860.50

46. Santos NC, Costa PS, Cunha P et al (2014) Clinical, physical and lifestyle variables and relationship with cognition and mood in aging: a cross-sectional analysis of distinct educational groups. Front Aging Neurosci 6:21

47. Smith E, Bailey PE, Crawford J et al (2014) Adiposity estimated using dual energy X-ray absorptiometry and body mass index and its association with cognition in elderly adults. J Am Geriatr Soc 62(12):2311-2318. https://doi.org/10.1111/jgs.13157

48. Kirton JW, Dotson VM (2016) The interactive effects of age, education, and BMI on cognitive functioning. Neuropsychol Dev Cogn B Aging Neuropsychol Cogn 23(2):253-262. https://doi.org/10.1080/ 13825585.2015.1082531

49. West RK, Ravona-Springer R, Schmeidler J et al (2014) The association of duration of type 2 diabetes with cognitive performance is modulated by long-term glycemic control. Am J Geriatr Psychiatry 22(10):1055-1059. https://doi.org/10.1016/j.jagp.2014.01.010

50. Yogi-Morren D, Galioto R, Strandjord SE et al (2014) Duration of type 2 diabetes and very low density lipoprotein levels are associated with cognitive dysfunction in metabolic syndrome. Cardiovasc Psychiatry Neurol 2014:656341. https://doi.org/10.1155/2014/ 656341

Publisher's note Springer Nature remains neutral with regard to jurisdictional claims in published maps and institutional affiliations. 